\title{
ON THE EXPONENTIAL STABILITY OF STOCHASTIC MARKOVIAN JUMP
}

\section{SYSTEMS}

\author{
E.K. Boukas, H. Yang
}

\begin{abstract}
-
This paper deals with the exponential stability of the class of stochastic systems with jumps. For the linear case, a sufficient condition, which guarantees that the nominal system with a bounded diffusion term remains stable under a state feedback control law, is established. For the case of uncertaiin linear stochastic system, we have designed an optimal control law that guarantees the robust exponential stability of the systems. Finally for the nonlinear case with matching conditions, we have established a similar result.
\end{abstract}

\section{INTRODUCTION}

For practical systems, we always recourse to the reduction of the model by making some assumptions that are in general not satisfied. The model we used is not a good representation of the system we want to control. Therefore it is necessary to consider the effect of the uncertainties if we want to guarantee that the real system remains at least stable. The knowledge of the dynamics or certain information on the uncertainties of the system under consideration is then an important point in the design phase.

Our goal is to design a control law that guarantees to the stochastic systems under study to remain exponentially stable when certain conditions for the uncertain terms and the perturbation terms are satisfied.

\section{Stability of Linear System}

In this section, we consider the class of jump linear systems described by the following stochastic differential equation:

$$
\begin{gathered}
d \mathbf{x}(t)=\mathbf{A}(\mathbf{r}(t)) \mathbf{x}(t) d t+\mathbf{B}(\mathbf{r}(t)) \mathbf{u}(t) d t \\
\quad+\mathbf{g}(\mathbf{x}(t), \mathbf{r}(t), t) d \mathbf{w}(t), \mathbf{x}(0)=\mathbf{x}_{0},
\end{gathered}
$$

where the $n$-dimensional vector $\mathbf{x}(t) \in \mathbb{R}^{n}$ stands for the state of the system and the $m$-dimensional vector $\mathbf{u}(t) \epsilon$ $\mathbb{R}^{m}$ is the control law. Vector $\mathbf{w}(t)$ is a vector standard Brownian motion. The parameter $\mathbf{r}(t)$ represents a continuous time discrete-state Markov process taking values in a finite $\operatorname{set} \mathcal{B}=\{1,2, \ldots, s\}$ with transition probability $p_{i j}(\mathbf{r}(t)):=P\{\mathbf{r}(t+\Delta t)=j \mid \mathbf{r}(t)=i\}$ given by:

$$
p_{i j}(\mathbf{r}(t))=\left\{\begin{array}{l}
q_{i j} \Delta t+o(\Delta t), \quad \text { if } i \neq j \\
1+q_{i i} \Delta t+o(\Delta t), \text { if } i=j
\end{array}\right.
$$

Mechanical Engineering Department, École Polytechnique de Montréal, P.O. Box 6079, Station "centre-ville", Montréal, Que., Canada, H3C 3A7. Research of this author was supported by the Natural Sciences and Engineering Research Council of Canada under grants OGP0036444. Email: Boukas@anas.meca.polymtl.ca

Department of Statistics, The University of Hong Kong, Hong Kong. Research of this author was supported by CRCG Grant C337/017/0019, The University of Hong Kong. Email: hlyang@husua.hku.hk.
In this relation, $q_{i j}$ stands for the transition probability rate from mode $i$ to mode $j$ and satisfies the following relations $q_{i j} \geq 0$ and $q_{i i}=-\sum_{j \in \mathcal{B}, i \neq j} q_{i j}$.

In Eq. (1), $\mathbf{A}(\mathbf{r}(t)), \mathbf{B}(\mathbf{r}(t)$ and $\mathbf{g}(\mathbf{x}(t), \mathbf{r}(t), t)$ are appropriately dimensioned matrices. The matrices $\mathbf{A}(\mathbf{r}(t))$ and $\mathbf{B}(\mathbf{r}(t)$ are constant for a given value of $\mathbf{r}(t) \cdot \mathbf{g}(\mathbf{x}(t), \mathbf{r}(t), t)$ is Lipschitz continuous for $\mathbf{x}$ and satisfying the linear growth condition. We will also assume that the Brownian motion $\mathbf{w}(t)$ and the jump process $\mathbf{r}(t)$ are independent.

In the remainder of this paper, we assume that the first component of the state vector, i.e. $\mathbf{x}(t)$ in each mode is available for feedback. Moreover the stochastic observability and controllability are assumed. In this section, we will use the technique given by Mao (Ref. 9) to show the exponential stochastic stability of the system described by Eqs. (1)-(2) when the state feedback control law is used.

Theorem 2.1: If the system without the Brownian motion terms is exponentially stable under the feedback control given by Eq. (7) and there is a nonnegative constant $\alpha$ such that:

$$
\begin{aligned}
& \max _{i}\left\{\operatorname{trace}\left[\mathbf{g}^{\prime}(\mathbf{x}(t), i, t) \mathbf{g}(\mathbf{x}(t), \mathbf{r}(t), t)\right\} \leq \alpha\|\mathbf{x}(t)\|^{2}(3)\right. \\
& \min _{i}\left[\lambda_{\min }(\mathbf{Q}(i)]>\alpha \max _{i}\|\mathbf{P}(i)\|\right.
\end{aligned}
$$

where $\lambda_{\min }(\mathbf{Q}(\mathbf{r}(t))$ is the smallest eigenvalue of $\mathbf{Q}(\mathbf{r}(t))$. Then the system (1)-(2) is exponentially stable in the mean square.

\section{Robust Stability of the Systems With UNCERTAIN COEFFICIENT}

Let the real system be described by the following stochastic differential equations:

$$
\begin{aligned}
& d \mathbf{x}(t)=[\mathbf{A}(\mathbf{r}(t))+\Delta \mathbf{A}(\mathbf{r}(t)] \mathbf{x}(t) d t+[\mathbf{B}(\mathbf{r}(t)) \\
& +\Delta \mathbf{B}(\mathbf{r}(t))] \mathbf{u}(t) d t+\mathbf{g}(\mathbf{x}, \mathbf{r}(t), t) d \mathbf{w}(t), \mathbf{x}(0)=\mathbf{x}_{0}(5)
\end{aligned}
$$

where the matrices $\Delta \mathbf{A}(\mathbf{r}(t))$ and $\Delta \mathbf{B}(\mathbf{r}(t))$ represent respectively the uncertainties on the matrices $\mathbf{A}(\mathbf{r}(t))$ and $\mathbf{B}(\mathbf{r}(t))$ for each value of $\mathbf{r}(t), \mathbf{x}(t)$ and $\mathbf{u}(t)$ have the same meaning as previous.

Assumption 3.1 For each $i \in \mathcal{B}$, there are nonnegative symmetric matrices $\mathbf{Q}_{0}(i)$ and $\mathbf{R}_{0}(i)$ and positive scalars $\gamma_{a}(i)$ and $\gamma_{b}(i)$, such that:

$$
\begin{aligned}
& \Delta \mathbf{A}^{\prime}(\mathbf{r}(t)) \Delta \mathbf{A}(\mathbf{r}(t)) \leq \gamma_{a}(\mathbf{r}(t)) \mathbf{Q}_{0}(\mathbf{r}(t)) \\
& \Delta \mathbf{B}^{\prime}(\mathbf{r}(t)) \Delta \mathbf{B}(\mathbf{r}(t)) \leq \gamma_{b}(\mathbf{r}(t)) \mathbf{R}_{0}(\mathbf{r}(t))
\end{aligned}
$$

where the matrix inequality $\mathbf{M} \leq \mathbf{N}$ for symmetric matrices $\mathbf{M}$ and $\mathbf{N}$ means that $\mathbf{N}-\mathbf{M}$ is a nonnegative matrix. 
To control the system (5)-(2), we will now use the optimal approach by choosing the weighting cost matrices $\mathbf{Q}(i)$ and $\mathbf{R}(i)$ as following:

$$
\mathbf{Q}(i)=\mathbf{Q}_{0}(i)+\mathbf{Q}_{1}(i), \quad \mathbf{R}(i)=\mathbf{R}_{0}(i)+\mathbf{R}_{1}(i)
$$

where $\mathbf{Q}_{0}(i)$ and $\mathbf{R}_{0}(i)$ are the matrices defined in (6)-(7) and $\mathbf{Q}_{1}(i)$ and $\mathbf{R}_{1}(i)$ are known symmetric matrices with $\mathbf{Q}_{1}(i)$ positive semidefinite and $\mathbf{R}_{1}(i)$ positive definite.

Assumption 3.2 Assume that the nominal system without the Brownian motion terms is stable under the control law obtained by solving the optimal control problem, i.e. $\mathbf{u}(\mathbf{x}, i)=-\mathbf{R}^{-1}(i) \mathbf{B}^{\prime}(i) \mathbf{P}(i) \mathbf{x}(t)$, where the weighting matrices are chosen by Eq. (8).

Let $\gamma(i)$ and $\Gamma(i)$ be defined by:

$$
\begin{aligned}
\mathbf{\Gamma}(i) & =\mathbf{P}^{-1}(i)\left[\mathbf{Q}_{1}(i)+\mathbf{K}^{\prime}(i) \mathbf{R}_{1}(i) \mathbf{K}(i)\right] \mathbf{P}^{-1}(i) \\
\gamma(i) & =\gamma_{a}(i)+\gamma_{b}(i)
\end{aligned}
$$

In the following, we present a sufficient condition which guarantees the stabilizability of the system (5)-(2).

Theorem 3.1. If the nominal system without the Brownian motion terms is stable under the state feedback control law obtained by solving the optimal control problem with the weighting matrices are chosen by Eqs. (18)-(19), there exists an $\alpha$ such that the following conditions are satisfied:

$$
\begin{aligned}
& \gamma(i) \mathbf{I}<\boldsymbol{\Gamma}(i) \\
& \max _{i} \operatorname{trace}\left[\mathbf{g}^{\prime}(\mathbf{x}(t), i, t) \mathbf{g}(\mathbf{x}(t), i, t)\right] \leq \alpha\|\mathbf{x}(t)\|^{2} \\
& \alpha\left\|\max _{i} \mathbf{P}(i)\right\|<\min _{i} \lambda_{\min }[\mathbf{P}(i)[\mathbf{\Gamma}(i)-\gamma(i) \mathbf{I}] \mathbf{P}(i, \mid 13)
\end{aligned}
$$

Then the real system remains exponentially stable in the whole ranges of uncertainty.

\section{Stability of Nonlinear Systems}

We consider an uncertain stochastic nonlinear system described by:

$$
\begin{aligned}
& d \mathbf{x}(t)=[\mathbf{A}(\mathbf{x}(t), \mathbf{r}(t), t)+\Delta \mathbf{A}(\mathbf{x}(t), \mathbf{r}(t), t)] d t \\
& \quad+[\mathbf{B}(\mathbf{x}(t), \mathbf{r}(t), t)+\Delta \mathbf{B}(\mathbf{x}(t), \mathbf{r}(t), t)] \mathbf{u}(t) d t \\
& \quad+\mathbf{g}(\mathbf{x}(t), \mathbf{r}(t), t) d \mathbf{w}(t)
\end{aligned}
$$

where the $n$-dimensional vector $\mathbf{x}(t) \in \mathbb{R}^{n}$ stands for the state of the system and the m-dimensional vector $\mathbf{u}(t) \in$ $\mathbb{R}^{m}$ is the control law. $\mathbf{A}(\mathbf{x}, \mathbf{r}, t), \Delta \mathbf{A}(\mathbf{x}, \mathbf{r}, t), \mathbf{B}(\mathbf{x}, \mathbf{r}, t)$, $\Delta \mathbf{B}(\mathbf{x}(t), \mathbf{r}(t), t)$ and $\mathbf{g}(\mathbf{x}(t), \mathbf{r}(t), t)$ are matrices of appropriate dimensions. $\mathbf{r}(t)$ is same as before. We will assume that all the matrices of the system are Lipschitz continuous functions and satisfying the linear growth condition. So the equation (14) has a unique solution.

Assumption 4.1. For each $i \in \mathcal{B}, \mathbf{A}(0, i, t)=0$, $\mathbf{B}(0, i, t)=0, \Delta \mathbf{A}(0, i, t)=0, \Delta \mathbf{B}(0, i, t)=0$ and $\mathrm{g}(0, i, t)=0$, for all $t \in \mathbb{R}$, and $\mathbf{x}=0$ is the unique equilibrium of (29) when $\mathbf{u}(t)=0$.

Assumption 4.2. For each mode $i \in \mathcal{B}$, there are mappings $\mathbf{D}(\mathbf{x}, i, t)$ and $\mathbf{E}(\mathbf{x}, i, t)$ such that

$$
\begin{aligned}
\Delta \mathbf{A}(\mathbf{x}, i, t) & =\mathbf{B}(\mathbf{x}, i, t) \mathbf{D}(\mathbf{x}, i, t) \\
\Delta \mathbf{B}(\mathbf{x}, i, t) & =\mathbf{B}(\mathbf{x}, i, t) \mathbf{E}(\mathbf{x}, i, t) \\
\|\mathbf{E}(\mathbf{x}, i, t)\| & <1
\end{aligned}
$$

where $\mathbf{D}($.$) and \mathbf{E}($.$) are matrices of appropriate dimensions$ which are continuous in $(\mathbf{x}, t)$ for each $i \in \mathcal{B}$.

Let the stochastic Lyapunov function $V(\mathbf{x}, i)$ be defined by: $V(\mathbf{x}, i)=\mathbf{x}^{\prime} \mathbf{P}(i) \mathbf{x}$, where $\mathbf{P}(i)$ is a symmetric positive definite matrix.

Assumption 4.3. Let $A_{0} V(i, \mathrm{x})$ be defined by:

$$
A_{0} V(\mathbf{x}, i)=\nabla_{x}^{\prime} V(\mathbf{x}) \mathbf{A}(\mathbf{x}, i, t)+\sum_{j \in \mathcal{B}} q_{i j} V(\mathbf{x}, j)
$$

where $\nabla_{x}^{\prime}$ denotes the transpose of gradient operation. We also require that there is a constant $\gamma_{1}>0$ such that

$$
A_{0} V(\mathbf{x}, i) \leq-\gamma_{1} V(\mathbf{x}, i)
$$

In the following, we use the same technique to construct a control law $\mathbf{u}^{*}($.$) which will later be shown to stabilize$ the class of systems under study. The first step in the construction of the control law is to select two functions $\Delta_{1}($.$) and \Delta_{2}($.$) , independent of the uncertainty such that:$

$$
\begin{aligned}
\|\mathbf{D}(\mathbf{x}, i, t)\| & \leq \Delta_{1}(\mathbf{x}, i, t) \\
\|\mathbf{E}(\mathbf{x}, i, t)\| & \leq \Delta_{2}(\mathbf{x}, i, t)
\end{aligned}
$$

Now, for each mode $i \in \mathcal{B}$, we choose any nonnegative function $\gamma(\mathbf{x}, i, t)$ which is continuous in $(\mathbf{x}, t)$ and satisfying the following inequality:

$$
\gamma(\mathbf{x}, i, t) \geq-\frac{\Delta_{1}^{2}(\mathbf{x}, i, t)}{4 C_{1}\left[A_{0} V(\mathbf{x}, i)+1\right]\left[1-\Delta_{2}(\mathbf{x}, i, t)\right]}
$$

where $0<C_{1}<1$.

We define the controller by the following expression:

$$
\mathbf{u}^{*}(\mathbf{x}, i, t):=-\gamma(\mathbf{x}, i, t) \mathbf{B}^{\prime}(\mathbf{x}, i, t) \nabla_{x} V(\mathbf{x}, i)
$$

The following result states the sufficient condition which guarantee the exponential stability of the class of nonlinear system (14).

Theorem 4.1. Assume that the assumptions 4.1-4.3 are satisfied and the controller constructed above is used. Assume also that

$$
\begin{aligned}
& \max _{i} \operatorname{trace}\left[\mathbf{g}^{\prime}(\mathbf{x}(t), \mathbf{r}(t), t) \mathbf{g}(\mathbf{x}(t), \mathbf{r}(t), t)\right] \leq \alpha \\
& \min _{i}\left[\lambda_{\min }[\mathbf{P}(i)]\right] \gamma_{1}>\alpha \max _{i}\|\mathbf{P}(i)\|
\end{aligned}
$$

are verified. Then the uncertain nonlinear system (14) is exponentially stable.

\section{REFERENCES}

1. Boukas, E.K. and Yang, H.: "Robust Stability of Nonlinear Piecewise Deterministic Systems under Matching Conditions", Mathematical Problems in Engineering: Theory, Method, and Applications, to appear 1997.

2. Mao, X. "Robustness of Exponential Stability of Stochastic Differential Delay Equations", IEEE Trans. Autom. Contr., Vol. AC-41, No. 3, (1996), 442 - 447.

3. Neto, A.T., Dion, J.M. and Dugard, L.(1992). Robustness Bounds for LQ Regulators. IEEE Trans. Autom. Contr., Vol. 37, No. 9, pp. 1373 - 1377. 